\title{
In Situ Observation of Atomic Redistribution in Alloying Gold-Silver Nanorods
}

Jessi E. S. van der Hoeven, ${ }^{\dagger,}{ }^{+}$Tom A. J. Welling, ${ }^{\ddagger}$ Tiago A. G. Silva, ${ }^{\S}$ Jeroen E. van den Reijen, ${ }^{\dagger}$ Camille La Fontaine," Xavier Carrier, ${ }^{\S}$ Catherine Louis, ${ }^{\S}$ Alfons van Blaaderen, ${ }^{*}$, and Petra E. de Jongh* ${ }^{* \dagger}+$ (i)

${ }^{\dagger}$ Inorganic Chemistry and Catalysis, Debye Institute for Nanomaterials Science, Utrecht University, Universiteitsweg 99,3584 CG Utrecht, The Netherlands

${ }^{*}$ Soft Condensed Matter, Debye Institute for Nanomaterials Science, Utrecht University, Princetonplein 5, 3584 CC Utrecht, The Netherlands

${ }^{\S}$ Laboratoire de Réactivité de Surface, Sorbonne Université, CNRS, F-75005, Paris, France

"L’Orme des Merisiers, Synchrotron SOLEIL, BP 48, Saint-Aubin, 91192 Gif-sur-Yvette, France

\section{Supporting Information}

ABSTRACT: The catalytic performance and optical properties of bimetallic nanoparticles critically depend on the atomic distribution of the two metals in the nanoparticles. However, at elevated temperatures, during light-induced heating, or during catalysis, atomic redistribution can occur. Measuring such metal redistribution in situ is challenging, and a single experimental technique does not suffice. Furthermore, the availability of a well-defined nanoparticle system has been an obstacle for a systematic investigation of the key factors governing the atomic redistribution. In this study, we follow metal redistribution in precisely tunable, single-crystalline Au-core, Ag-shell nanorods in situ, both at a single particle and an ensemble-averaged level, by combining

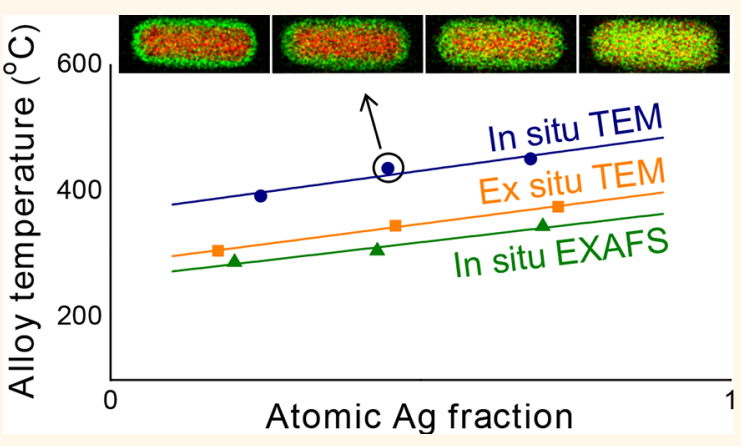
in situ transmission electron spectroscopy with in situ extended X-ray absorption fine structure validated by $e x$ situ measurements. We show that the kinetics of atomic redistribution in $\mathrm{Au}-\mathrm{Ag}$ nanoparticles depend on the metal composition and particle volume, such that a higher Ag content or a larger particle size led to significantly slower metal redistribution. We developed a simple theoretical model based on Fick's first law that can correctly predict the composition- and size-dependent alloying behavior in $\mathrm{Au}-\mathrm{Ag}$ nanoparticles, as observed experimentally.

KEYWORDS: alloying, bimetallics, in situ electron microscopy, in situ EXAFS, modeling

$\mathrm{B}$ $y$ the combination of two metals in bimetallic nanoparticles (NPs), new and enhanced optical and catalytic properties can arise that can lead to applications in, e.g., sensing, biomedicine, data storage, and catalysis. $^{1-10}$ The physicochemical properties of these bimetallic particles can be tuned not only by varying the metal composition but also by changing the atomic distribution of the two metals within the nanoparticles at a fixed composition (for example from core-shell to alloyed NPs). ${ }^{8,10-15}$ The exact atomic distribution of the metals is particularly important in catalysis, in which the atoms close to the surface play a dominant role in the catalytic performance. ${ }^{7,16-19}$ Furthermore, when exposing bimetallic nanoparticles to various gas atmospheres and heating them to elevated temperatures, atomic redistribution can occur. $^{11,17,18,20-26}$ This alters the optical ${ }^{8,13,26}$ and catalytic properties $^{16-18,24,27}$ and can even lead to severe deactivation of the catalyst. Therefore, understanding atomic restructuring is crucial in the design of new catalytic and optical bimetallic materials.

Various techniques have been employed to follow metal redistribution in situ, each providing information on a different length scale. ${ }^{20}$ Single-particle studies often make use of in situ transmission electron microscopy (TEM). With this technique, sub-nanometer or even atomic resolutions can be obtained while heating the sample. ${ }^{17,21,22}$ This technique, however, is limited to samples that are very stable under electron irradiation to avoid electron-beam-induced artifacts. ${ }^{28-30}$ Therefore, to verify the influence of the electron beam, it is important to also perform ex situ heating measurements. ${ }^{30}$ Alternatively, X-ray-based techniques, such as X-ray photo-

Received: May 26, 2018

Accepted: July 11, 2018

Published: July 16, 2018 
electron spectroscopy (XPS) and X-ray absorption fine structure (XAFS), also offer atomic information but averaged over a much larger number of particles. ${ }^{18,25,31}$ XPS allows us to specifically study the surface composition of the NPs, and it is thus particularly suitable to measure surface segregation effects. ${ }^{17,23,24}$ However, XAFS measurements give insight in the degree of mixing and oxidation state of the atoms within the nanoparticles and can be carried out in different gas atmospheres. ${ }^{18,25,31}$ Thus, to follow the metal redistribution in bimetallic nanoparticles at multiple length scales (on an atomic, single-particle, and ensemble-averaged level), one technique does not suffice, and a multi-technique approach is required.

In addition, a systematic, quantitative, and reproducible study of atomic restructuring requires a well-defined model system. The use of rather heterogeneous bimetallic catalysts, obtained via standard catalyst preparation methods, is especially problematic when using techniques such as XAFS and XPS, in which the measured signal is an ensemble average. Therefore, the influence of fundamental parameters such as the metal composition and particle volume on the atomic redistribution process in bimetallic nanoparticles are largely unexplored.

In this study, we investigated the thermally driven atomic redistribution in single crystalline $\mathrm{Au}-\mathrm{Ag}$ core-shell nanorods in situ both on a single particle and an ensemble-averaged level. We employed colloidally synthesized Au-core, Ag-shell nanorods of which the composition, size, and shape was tuned precisely. ${ }^{9}$ By the coating of the metal nanorods with a protective mesoporous silica coating, ${ }^{32}$ preservation of the particle shape during atomic redistribution was ensured. ${ }^{8}$ We specifically chose a $\mathrm{Au}-\mathrm{Ag}$-based system because alloy formation is thermodynamically favorable at all compositions and the lattice spacings of $\mathrm{Au}$ and $\mathrm{Ag}$ closely match. ${ }^{33}$ Because the nanorods are single-crystalline, this model system is wellsuited to specifically study the kinetics of metal redistribution during alloying. To this end, we performed both in situ TEM and in situ EXAFS measurements, yielding sub-nanometer, single-particle and atomic, ensemble-averaged information, respectively. In addition, we validated the in situ measurements with ex situ measurements carried out in the absence of an electron or X-ray beam. In particular, we addressed the influence of the metal composition $(\mathrm{Au}-\mathrm{Ag}$ ratio) on the alloying temperature of the Au-core, Ag-shell nanorods. We unambiguously showed the influence of the metal composition on the kinetics of the alloying process. Increasing Ag content led to slower metal redistribution, a trend that is opposite to the dependence of the melting temperature on the $\mathrm{Au}-\mathrm{Ag}$ ratio. In addition, indications for size-dependent alloying were found where a decrease in particle volume led to lower alloying temperatures. We developed a simple theoretical model that correctly predicts the temperatures and time scales for metal redistribution as a function of particle volume and composition. Our study not only demonstrates a general, multiscale approach to monitoring metal redistribution in bimetallic nanoparticles but also reveals the influence of fundamental parameters governing metal redistribution, which is of importance in bimetallic nanoparticle applications.

\section{RESULTS AND DISCUSSION}

Preparation of Core-Shell Nanorods. Mesoporous silica-coated Au-core, Ag-shell nanorods (Au@Ag@SiO $\mathrm{NRs)}$ with similar volumes but with three different Au-to-Ag ratios were colloidally synthesized. The colloidal synthesis was performed on a relatively large (milligram) scale to obtain the required amount of sample needed for the EXAFS measurements. To this end, the Ag-shell growth as described by Deng et al., comprising the reduction of $\mathrm{Ag}^{+}$ions on the $\mathrm{Au}$ nanorods by ascorbic acid, was performed in an acidified, instead of neutral, aqueous solution. ${ }^{9}$ The presence of $\mathrm{H}^{+}$ions slowed the Ag-shell growth down considerably (from seconds to minutes), resulting in sufficiently long mixing times for the reagents and homogeneous Ag-shell growth. To limit the variation in particle volume when changing the Au-to-Ag ratios of the particles, both the core and the shell size of the Au core and $\mathrm{Ag}$ shell were varied. In this way, 3 batches of mesoporous silica coated $\mathrm{Au}$-core $\mathrm{Ag}$-shell $\mathrm{NRs}$ with an average atomic $\mathrm{Ag}$ fraction $X_{\mathrm{Ag}}$ of $0.20,0.46$, and 0.70 and an average particle volume $V$ of $2.2,4.1$, and $5.6 \times 10^{4} \mathrm{~nm}^{3}$, respectively, were obtained. To also investigate the influence of the particle volume on the atomic redistribution, a batch of considerably smallerAu@Ag@SiO 2 NRs with an average of $X_{\mathrm{Ag}}=0.46$ and $V=0.7 \times 10^{4} \mathrm{~nm}^{3}$ was prepared.

In Table 1, we report a summary of the sample details, and in Figure 1, we show the corresponding high annular dark-field

Table 1. Sample Details for the Au@Ag@SiO ${ }_{2}$ NRs Depicted in Figure $1^{a}$

$\begin{array}{llll}X_{\mathrm{Ag}} & L(\mathrm{~nm}) & D(\mathrm{~nm}) & V \cdot 10^{4}\left(\mathrm{~nm}^{3}\right) \\ 0.20 & 67 \pm 10 & 21 \pm 2.1 & 2.2 \pm 0.58 \\ 0.46 & 74 \pm 8.7 & 28 \pm 1.9 & 4.1 \pm 0.77 \\ 0.70 & 80 \pm 9.2 & 32 \pm 3.8 & 5.6 \pm 1.6 \\ 0.46 & 48 \pm 9.2 & 14 \pm 1.8 & 0.7 \pm 0.3\end{array}$

${ }^{a}$ The average and corresponding standard deviations of the atomic $\mathrm{Ag}$ fraction, length, diameter, and volume are indicated with $X_{\mathrm{Ag}}, L, D$, and $V$, respectively. The values were based on 50 measurements per sample.

scanning transmission electron microscopy (HAADF-STEM) images and energy dispersive X-ray spectroscopy (EDX) maps. Due to the large $Z$ contrast difference between $\mathrm{Au}$ and $\mathrm{Ag}$ atoms, the core-shell structure of the nanorods is readily visible in the HAADF-STEM images. The different $\mathrm{Ag}$ contents are most clearly seen in the EDX maps, in which $\mathrm{Au}$ and $\mathrm{Ag}$ are depicted in red and green, respectively. The Si signal of the silica shell is shown in Figure S1 together with the optical spectra (Figure S2) and a high-resolution TEM image showing the single crystalline structure of the nanorods (Figure S3).

Direct Visualization of Metal Redistribution in Individual Particles with in Situ TEM. In situ TEM was used to visualize the atomic redistribution in individual NRs with different Au-to-Ag ratios and volumes. To avoid variations between in situ TEM measurements on different samples due to, e.g., inequalities in the heating temperature or differences in electron dose that are known to be important in in situ electron microscopy, ${ }^{28-30}$ we chose to compare four different samples in one measurement under exactly the same conditions. To achieve this, a mixture of the four samples with different Au-to$\mathrm{Ag}$ ratios and particle volumes was deposited on a single $\mathrm{SiN}_{x}$ chip. The heating experiment was carried out in a high vacuum with a ramp of $3{ }^{\circ} \mathrm{C} / \mathrm{min}$. EDX analysis was used to map the $\mathrm{Au}$ and $\mathrm{Ag}$ metal distribution as a function of temperature.

Figure 2a shows the EDX maps of the mixture of Au@Ag@ $\mathrm{SiO}_{2} \mathrm{NRs}$ at various temperatures. The EDX maps of the 

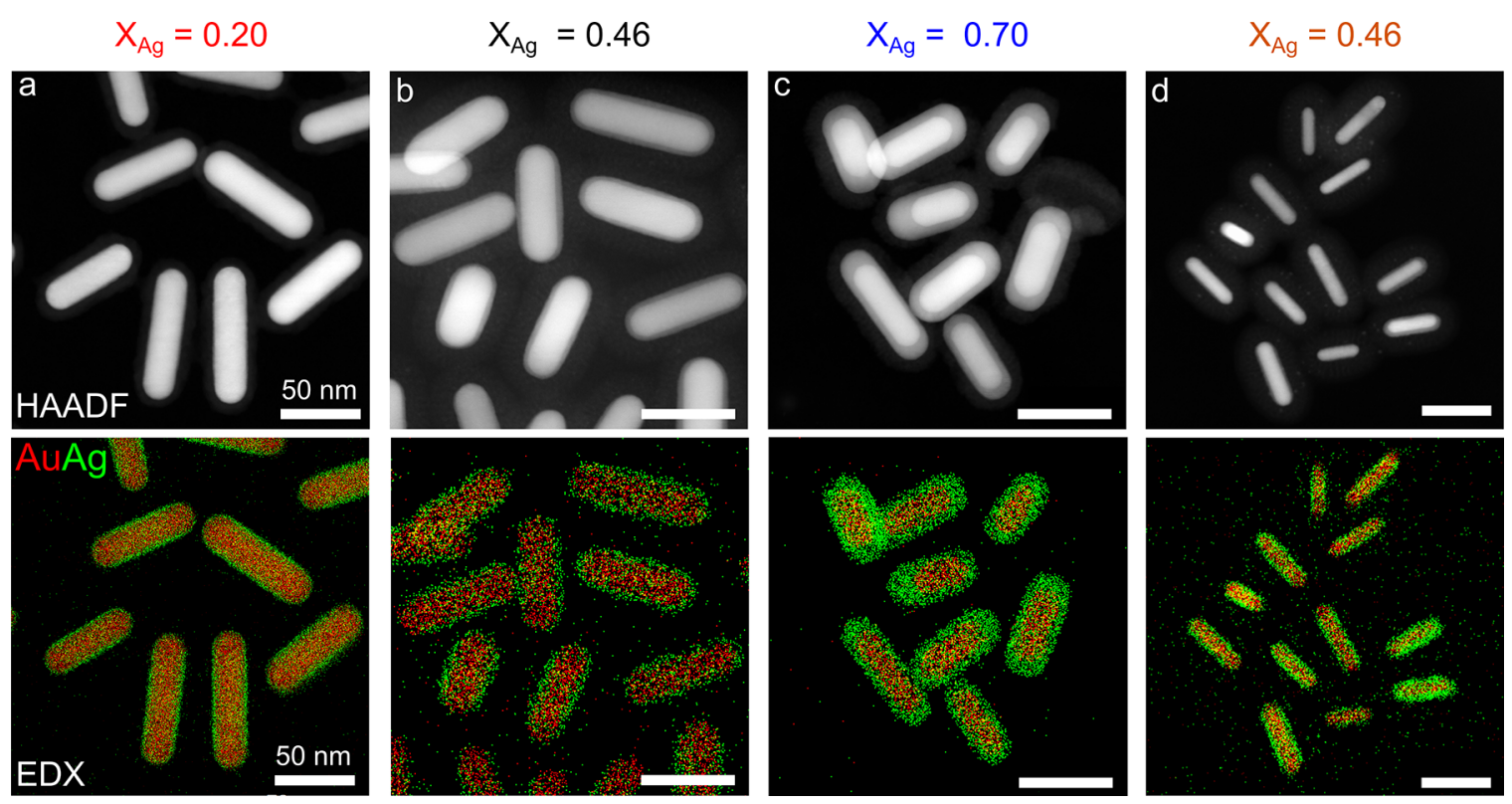

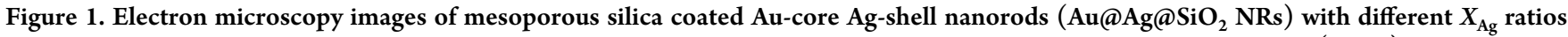
and particle volumes. Top: HAADF-STEM images. Bottom: EDX maps with Au and Ag in red and green, respectively. (a, red) Au@Ag@SiO NRs with $X_{\mathrm{Ag}}=0.20, V=2.2 \times 10^{4} \mathrm{~nm}^{3}$; (b, black) $X_{\mathrm{Ag}}=0.46, V=4.1 \times 10^{4} \mathrm{~nm}^{3}$; $(\mathrm{c}$, blue $) X_{\mathrm{Ag}}=0.70, V=5.6 \times 10^{4} \mathrm{~nm}^{3}$; and $(\mathrm{d}$, orange) $X_{\mathrm{Ag}}=0.46, V=0.7 \times 10^{4} \mathrm{~nm}^{3}$. The Si signal is not shown in the EDX maps (see Figure S1).

orange, red, gray, and blue highlighted NRs in Figure $2 \mathrm{a}$ are enlarged in Figure 2b. We determined the Ag fractions and particle volumes of these individual nanorods, which were slightly different from the average values in Table 1: $X_{\mathrm{Ag}}=0.44$ $V=0.45 \times 10^{4} \mathrm{~nm}^{3}$ (orange), $X_{\mathrm{Ag}}=0.45 \mathrm{~V}=5.0 \times 10^{4} \mathrm{~nm}^{3}$ (black), $X_{\mathrm{Ag}}=0.24 \mathrm{~V}=3.0 \times 10^{4} \mathrm{~nm}^{3}$ (red), and $X_{\mathrm{Ag}}=0.68 \mathrm{~V}$ $=2.7 \times 10^{4} \mathrm{~nm}^{3}$ (blue). To precisely track the metal redistribution in these individual nanorods during the heating process, we determined the core-to-shell ratio from the core and shell diameter for each particle at each temperature (see Figure S4 for details on the analysis procedure). From the core-to-shell ratios we derived the degree of alloying at the different heating temperatures, which increases from 0 to 1 when going from a core-shell to an alloyed nanorod. In Figure $2 c$, we plot the alloying curves of the black and orange highlighted single particles as a function of temperature for the 2 particles with the same $\mathrm{Au}-\mathrm{Ag}$ ratio but have a differing particle volume by a factor of 10 . The plot in Figure $2 \mathrm{~d}$ shows the individual alloying curves of the particles in red, black, and blue, which have a similar volume but different $\mathrm{Au}$-to-Ag ratios. We defined the alloying temperature $T_{\text {alloy }}$ as the temperature at which the degree of alloying reached 0.5 , which was 392 , 394,436 , and $451{ }^{\circ} \mathrm{C}$ for the rods with $X_{\mathrm{Ag}}=0.24,0.44,0.45$, and 0.68 , respectively.

These in situ TEM measurements clearly show the impact of the particle volume and the metal composition on the atomic redistribution, where a decrease in particle volume and $\mathrm{Ag}$ content led to significantly lower alloying temperatures. Lowering of the particle volume by a factor 10 (from $V=$ $5.0 \times 10^{4} \mathrm{~nm}^{3}$ to $0.45 \times 10^{4} \mathrm{~nm}^{3}$ ) resulted in a decrease in alloying temperature of $42{ }^{\circ} \mathrm{C}$, whereas the influence of the particle volume for larger NRs with $V=1.3$ to $3.0 \times 10^{4} \mathrm{~nm}^{3}$ was negligible (Figure S5). The considerable drop in the alloying temperature when decreasing the particle volume to $V$ $=0.45 \times 10^{4} \mathrm{~nm}^{3}$, and the diameter of the nanorod below 20 $\mathrm{nm}$ indicates an increased atom mobility at smaller particle dimensions. Such a size effect is in line with the previously reported particle-size-dependent melting of silica encapsulated AuNPs, in which the melting point decreased drastically from $\sim 900$ to $300{ }^{\circ} \mathrm{C}$ when decreasing the (spherical) particle diameter from 20 to $1.5 \mathrm{~nm}^{34}$

From EDX maps and corresponding alloying curves in Figure $2 \mathrm{~b}, \mathrm{~d}$, it is clear that the atomic redistribution is also strongly influenced by the $\mathrm{Au}-\mathrm{Ag}$ ratio: the $\mathrm{Au} @ \mathrm{Ag} @ \mathrm{SiO}_{2}$ NR with the $X_{\mathrm{Ag}}=0.68$ alloyed at almost $50{ }^{\circ} \mathrm{C}$ higher than the one with $X_{\mathrm{Ag}}=0.24$. Despite the fact that the rod with $X_{\mathrm{Ag}}$ $=0.68$ had a volume 2 times smaller than that of the rod with $X_{\mathrm{Ag}}=0.45$, the increase in $\mathrm{Ag}$ content led to a significantly higher alloying temperature.

Ensemble-Averaged Atomic Redistribution from in Situ EXAFS. To investigate the impact of the metal composition on the atomic redistribution for a larger number of particles, we moved from in situ TEM to in situ EXAFS and extended our study from a femtogram to a milligram scale and from a single particle to $10^{19}$ particles. Additionally, in situ EXAFS measurements allowed the dosing of gases combined with a reliable temperature control. The unconventionally fast switching between the metal absorption edges $(<1 \mathrm{~min})$ at the ROCK beamline of the SOLEIL synchrotron made it possible to follow the atomic redistribution at the $\mathrm{Au}$ and $\mathrm{Ag}$ absorption edges in the same experiment. The alloying experiments were carried out under inert conditions in a He flow because the presence of oxygen is known to significantly change the alloying process.

The in situ EXAFS data of the atomic redistribution in the $\mathrm{Au} @ \mathrm{Ag} @ \mathrm{SiO}_{2} \mathrm{NRs}$ with the lowest and highest Ag content, $X_{\mathrm{Ag}}=0.20$ and 0.70 , are shown in Figure 3. Figure $3 \mathrm{a}-\mathrm{d}$ shows the normalized $\mu(E)$ spectra and $\chi(k)$ spectra acquired at the $\mathrm{Au} \mathrm{L}_{3}$ and $\mathrm{Ag} \mathrm{K}$ absorption edges of the NRs with $X_{\mathrm{Ag}}=0.70$. The oxidation state of the $\mathrm{Au}$ and $\mathrm{Ag}$ atoms in the core and in the shell of the NRs before heating was determined from the XAFS spectra at room temperature (RT) and found to be predominately metallic (Figure S6). The in situ EXAFS spectra show a clear change when heating the NRs from 30 to $500{ }^{\circ} \mathrm{C}$. 
a
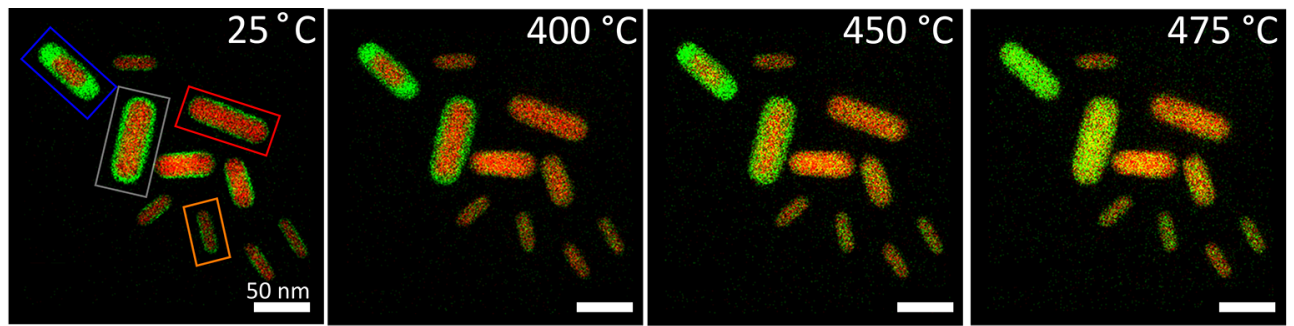

b
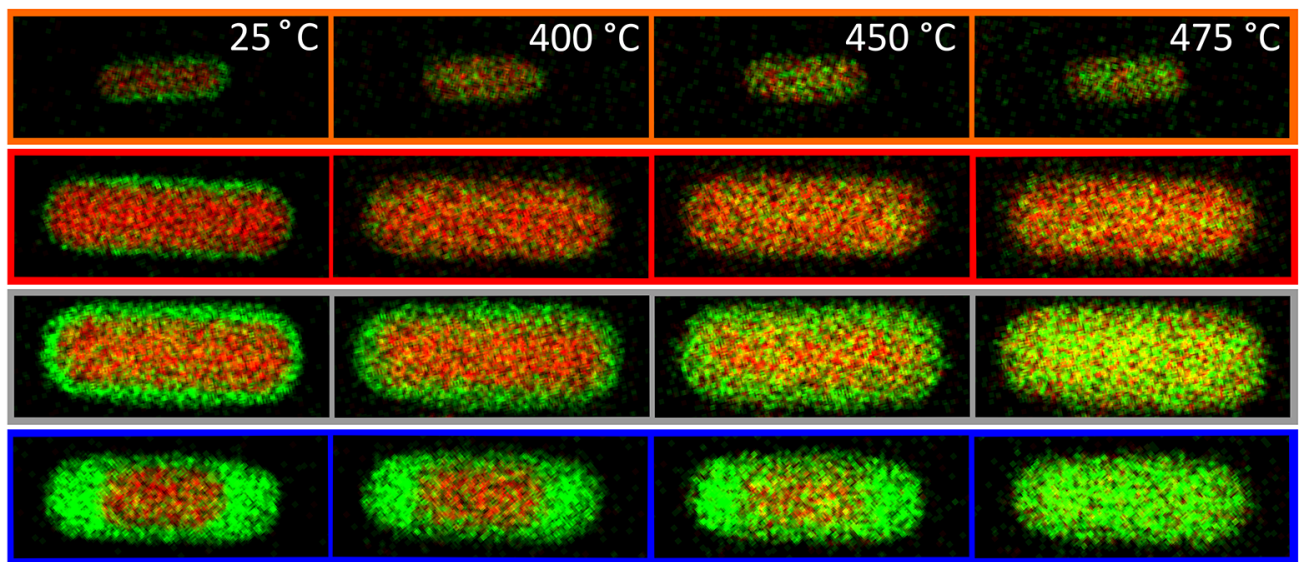

C

Volume dependence

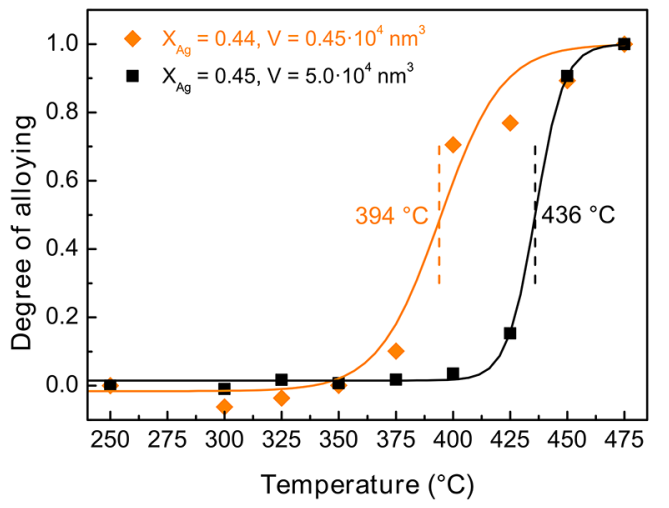

d

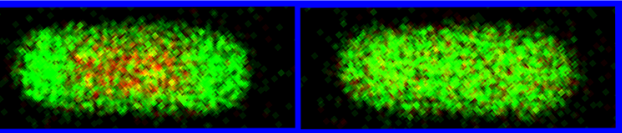

Au-Ag dependence

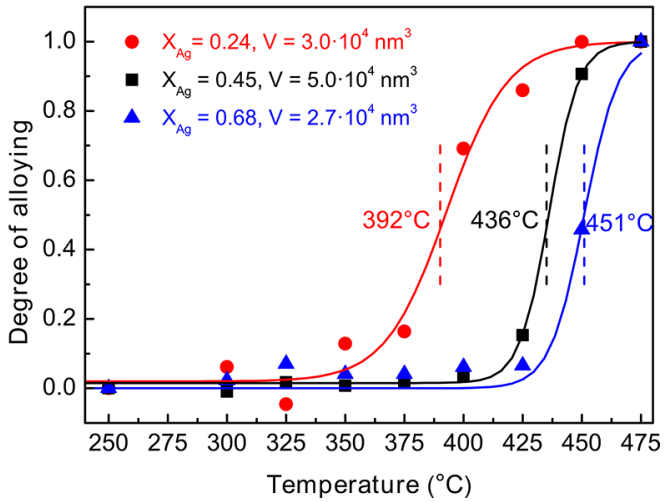

Figure 2. Direct visualization of atomic redistribution in individual $\mathrm{Au} @ \mathrm{Ag} @ \mathrm{SiO}_{2} \mathrm{NRs}$ with in situ heating TEM. (a, b) EDX maps acquired at 25, 400, 450, and $475^{\circ} \mathrm{C}$. (c) Particle volume dependence of the degree of alloying for $\mathrm{Au} @ \mathrm{Ag} @ S i \mathrm{O}_{2} \mathrm{NRs}$ with $V=0.45 \times 10^{4} \mathrm{~nm}^{3}\left(X_{\mathrm{Ag}}=\right.$ 0.44 , orange) and $V=5.0 \times 10^{4} \mathrm{~nm}^{3}\left(X_{\mathrm{Ag}}=0.45\right.$, black $)$. (d) The degree of alloying as a function of Ag-content with Au@Ag@SiO $\mathrm{NRs}_{2}$ $X_{\mathrm{Ag}}=0.24\left(V=3.0 \times 10^{4} \mathrm{~nm}^{3}\right.$, red $), X_{\mathrm{Ag}}=0.45\left(V=5.0 \times 10^{4} \mathrm{~nm}^{3}\right.$, black $)$, and $X_{\mathrm{Ag}}=0.68\left(V=2.7 \times 10^{4} \mathrm{~nm}^{3}\right.$, blue $)$. Curves are best fit to the experimental data. The heating ramp was set to $3{ }^{\circ} \mathrm{C} / \mathrm{min}$.

To verify if metal redistribution took place, we used the EXAFS spectra before and after thermal treatment to calculate the coordination numbers between the $\mathrm{Au}$ and $\mathrm{Ag}$ atoms: $N_{\mathrm{Au}-\mathrm{Au}}, N_{\mathrm{Au}-\mathrm{Ag}}, N_{\mathrm{Ag}-\mathrm{Ag}}$, and $N_{\mathrm{Ag}-\mathrm{Au}}$. Table 2 lists the coordination numbers for both samples. Due to the coreshell structure of the NRs, the coordination numbers between unlike atoms are low before heating. As expected, $N_{\mathrm{Ag}-\mathrm{Au}}$ is lowest for core-shell particles with the highest $X_{\mathrm{Ag}}$. After heating of the core-shell NRs to $500{ }^{\circ} \mathrm{C}, N_{\mathrm{Ag}-\mathrm{Au}}$ and $N_{\mathrm{Au}-\mathrm{Ag}}$ increased by a factor of $\geq 6$, indicating that mixing of the two elements took place in both samples. A full overview of the EXAFS fitting parameters is given in Tables S1-S4.

To estimate if the NRs were fully alloyed, meaning that the $\mathrm{Au}$ and $\mathrm{Ag}$ atoms were randomly dispersed within the particles, the extent of alloying $(J)$ was calculated following the approach developed by Hwang et al.: ${ }^{14}$

$$
J_{\mathrm{Au}}=\frac{P_{\text {observed }}}{P_{\text {random }}}=\frac{\left[N_{\mathrm{Au}-\mathrm{Ag}} /\left(N_{\mathrm{Au}-\mathrm{Ag}}+N_{\mathrm{Au}-\mathrm{Au}}\right)\right]_{\mathrm{observed}}}{\left[N_{\mathrm{Au}-\mathrm{Ag}} /\left(N_{\mathrm{Au}-\mathrm{Ag}}+N_{\mathrm{Au}-\mathrm{Au}}\right)\right]_{\text {random }}} \times 100 \%
$$

The $J$ values of the two components ( $\mathrm{Au}$ and $\mathrm{Ag}$ ) give information on the internal distribution of the two components. ${ }^{14}$ To calculate $P_{\text {random }}$ the Au-to-Ag ratios as determined by EDX were used. In Table 2, the $J$ values for the two different NR samples before and after heating to $500{ }^{\circ} \mathrm{C}$ are given. For both alloyed samples, the calculated $J_{\mathrm{Au}}$ and $J_{\mathrm{Ag}}$ values are close to 100 , indicating that the NRs are likely to have a fully alloyed structure when heating them to $500{ }^{\circ} \mathrm{C}$.

To deduce the evolution of the alloying process from all the spectra acquired between 30 and $500{ }^{\circ} \mathrm{C}$, we performed linear combination fitting on the normalized $\mu(E)$ spectra. In (E)XAFS analysis, linear combination fitting is typically used to determine and follow changes in the oxidation state of metal nanoparticles but is not common for following metal 

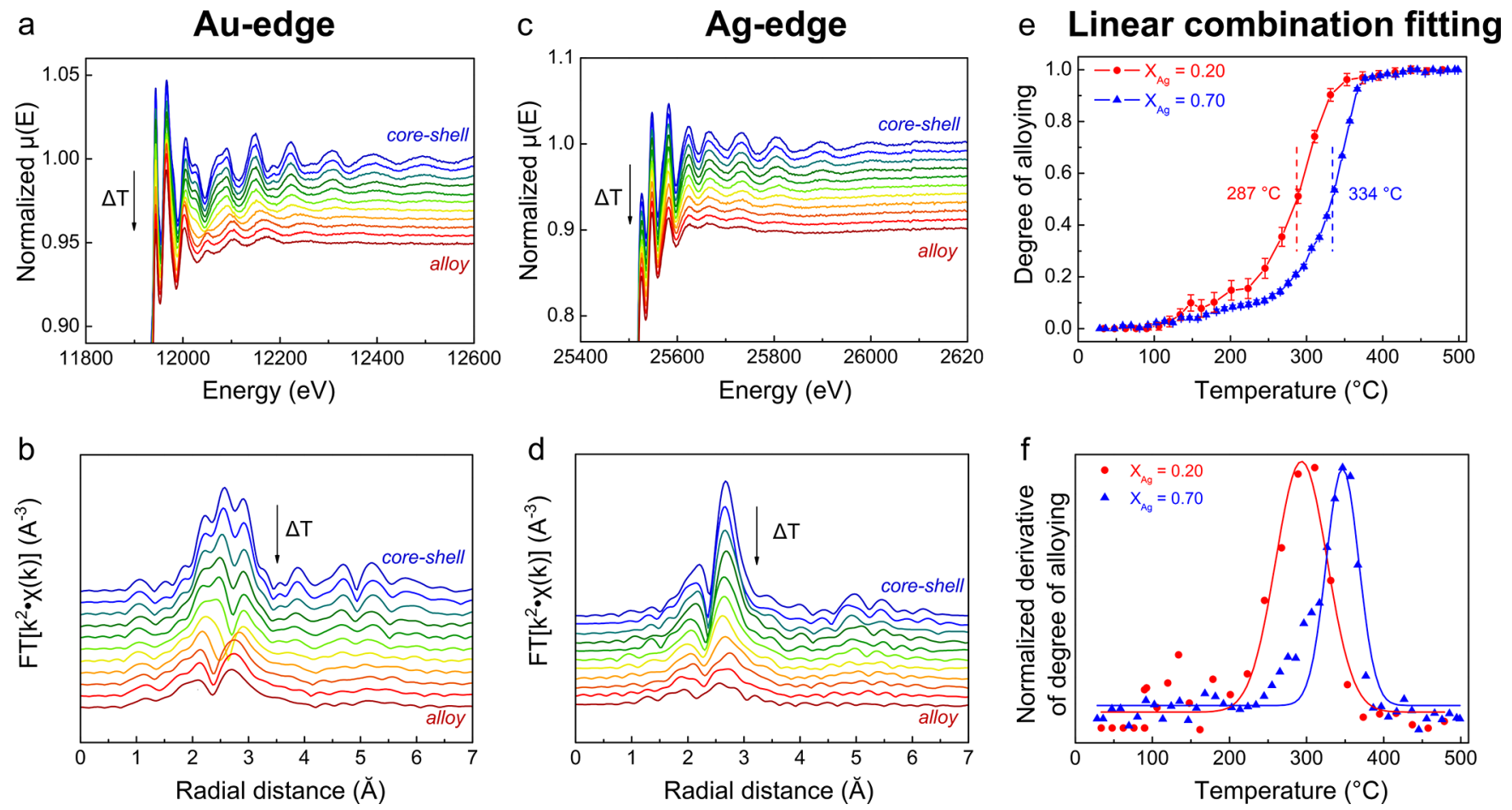

Figure 3. Double-edged in situ EXAFS measurements of $\mathrm{Au} @ \mathrm{Ag} @ \mathrm{SiO}_{2} \mathrm{NRs}$ upon heating. Normalized $\mu(E)$ spectra and FT $\left[k^{2} \chi(k)\right]$ spectra at the $A u L_{3}$ edge (panels a and $\mathrm{b}, \Delta k=3.3-14.0 \AA^{-1}$ ) and $\mathrm{Ag} \mathrm{K}$ edge (panels $\mathrm{c}$ and $\mathrm{d}, \Delta k=3.2-12.0 \AA^{-1}$ ) of the nanorods with $X_{\mathrm{Ag}}=0.70$, recorded every $\sim 50{ }^{\circ} \mathrm{C}$ when heating from 30 to $500{ }^{\circ} \mathrm{C}$. The plots in panels $e$ and $f$ show the degree of alloying and the derivative thereof as a function of temperature and were obtained by performing linear combination fitting on the normalized $\mu(E)$ spectra at the Ag K edge $\left(X_{\mathrm{Ag}}\right.$ $=0.20$, red $)$ and $\mathrm{Au} \mathrm{L}_{3}$ edge $\left(X_{\mathrm{Ag}}=0.70\right.$, blue $)$. The EXAFS spectra were acquired during heating to $500{ }^{\circ} \mathrm{C}$ with $3{ }^{\circ} \mathrm{C} / \mathrm{min}$ in a $25 \mathrm{~mL} / \mathrm{min}$ He flow.

Table 2. Coordination Number $N$ Before and After Heating the $\mathrm{NRs}$ to $500{ }^{\circ} \mathrm{C}$ in a $25 \mathrm{~mL} / \mathrm{min} \mathrm{He}$ Flow with a $3{ }^{\circ} \mathrm{C} / \mathrm{min}$ $\operatorname{Ramp}^{a}$

\begin{tabular}{|c|c|c|c|c|c|c|}
\hline & $N_{\mathrm{Ag}-\mathrm{Ag}}$ & $N_{\mathrm{Ag}-\mathrm{Au}}$ & $N_{\mathrm{Au}-\mathrm{Au}}$ & $N_{\mathrm{Au}-\mathrm{Ag}}$ & $J_{\mathrm{Ag}}$ & $J_{\mathrm{Au}}$ \\
\hline$X_{\mathrm{Ag}}=0.20$ before heating & $10.1 \pm 1.8$ & $1.1 \pm 1.3$ & $11.0 \pm 0.2$ & $0.3 \pm 0.2$ & 12 & 13 \\
\hline$X_{\mathrm{Ag}}=0.20$ after heating & $2.5 \pm 0.7$ & $6.8 \pm 1.7$ & $9.6 \pm 0.2$ & $1.8 \pm 0.1$ & 91 & 79 \\
\hline$X_{\mathrm{Ag}}=0.70$ before heating & $11.0 \pm 0.3$ & $0.6 \pm 0.3$ & $9.8 \pm 0.2$ & $0.3 \pm 0.2$ & 17 & 4 \\
\hline$X_{\mathrm{Ag}}=0.70$ after heating & $7.7 \pm 0.4$ & $3.5 \pm 0.2$ & $3.1 \pm 0.3$ & $8.3 \pm 0.4$ & 104 & 104 \\
\hline
\end{tabular}

${ }^{a}$ Based on the coordination numbers, the corresponding $J$ values were calculated.

redistribution. Note that a linear combination fitting based analysis is considerably faster than calculation of the coordination numbers, which is especially important when analyzing a large number of EXAFS spectra.

In our analysis, each EXAFS spectrum at a given temperature was compared to the spectrum of the initial core-shell and final alloyed state for which the spectra at 30 and $500{ }^{\circ} \mathrm{C}$ were taken, respectively. As shown in Figure 3e, the analysis was successfully applied to obtain the degree of alloying as a function of temperature. Figure $3 \mathrm{e}$ specifically shows the linear combination fitting results determined from the Ag K edge for the $X_{\mathrm{Ag}}=0.20$ sample and the $\mathrm{Au} \mathrm{L}_{3}$ edge for the $X_{\mathrm{Ag}}=0.70$ sample because the change from core-shell to alloyed state is most apparent on the edge of the least abundant metal. In addition, it is important to note that the linear combination fitting analysis is also sensitive to changes that are not due to metal redistribution, such as the damping of the EXAFS spectra due to thermal disorder with increasing temperature. This temperature contribution predominately plays a role when the change upon alloying is small, as is the case for the absorption edge of the metal that is in the majority (Figures S7 and S8).

From Figure $3 \mathrm{e}$, the alloying temperature determined at a degree of alloying of 0.5 , was 287 and $334{ }^{\circ} \mathrm{C}$ for the sample with $X_{\mathrm{Ag}}=0.20$ and 0.70 , respectively. The EXAFS measurements thus confirmed the increase in alloying temperature with increasing $\mathrm{Ag}$ content, as observed in the in situ TEM but now for a large ensemble of particles. However, it should be noted that there is a discrepancy in alloying temperatures: from the in situ EXAFS, we obtained $\sim 100{ }^{\circ} \mathrm{C}$ lower alloying temperatures compared with the in situ TEM data. This discrepancy demonstrates the need for ex situ measurements to establish the absolute temperature at which the metal redistribution occurs in the absence of an electron or $\mathrm{X}$-ray beam.

Validation of the in Situ Data. Although electron microscopy and X-ray absorption spectroscopy enable the in situ observation of structural changes in metal nanoparticles, it is crucial to validate these techniques with ex situ measurements. In particular, electron beam irradiation has been reported to induce anomalous behavior in nanostructured 
a

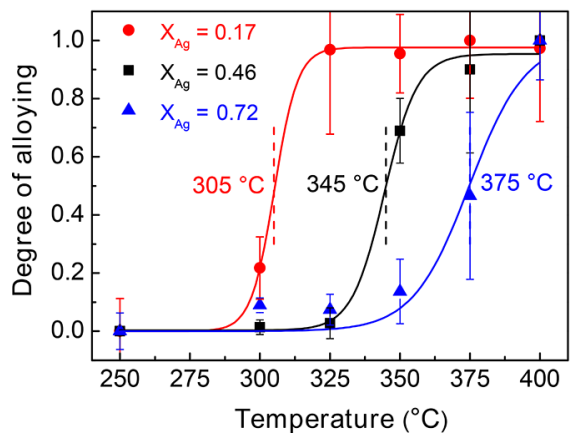

b All techniques

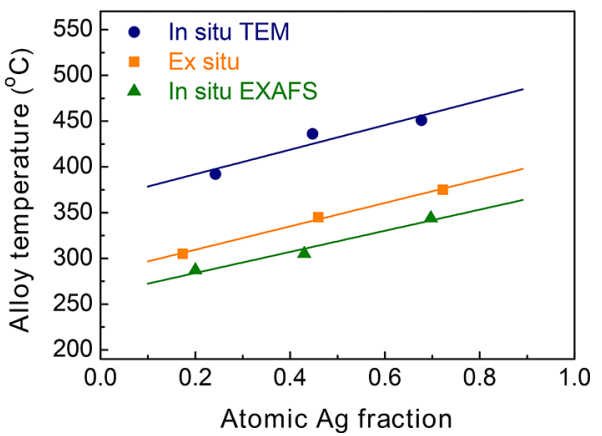

Figure 4. Ex situ TEM measurements on the alloying of $\mathrm{Au} @ \mathrm{Ag} @ \mathrm{SiO}_{2}$ NRs. (a) The degree of alloying after heating Au@Ag@SiO $\mathrm{NRs}_{2}$ in a furnace as a function of the heating temperature. Each point is an average of four particles. The alloying temperatures for the Au@Ag@SiO NRs with $X_{\mathrm{Ag}}=0.17$ (red), 0.46 (black), and 0.72 (blue) was 305,345 , and $375{ }^{\circ} \mathrm{C}$, respectively. The samples were heated in a $\mathrm{N}_{2}$ flow with a heating ramp of $3{ }^{\circ} \mathrm{C} / \mathrm{min}$. (b) A summary of the alloying temperature as a function of $\mathrm{Ag}$ fraction determined with in situ TEM (dark blue), $e x$ situ TEM (orange), and in situ EXAFS (green). Curves are best fit to the experimental data.

materials and significantly alter the deformation behavior, growth kinetics, and the structure of the nanoparticles during in situ studies. ${ }^{28-30}$ To verify the dependence of the alloying temperature on the Au-to-Ag ratio, as observed in in situ TEM and in situ EXAFS, ex situ measurements were carried out by heating the NRs in a furnace. Herein, we used the same heating ramp of $3{ }^{\circ} \mathrm{C} / \mathrm{min}$ to heat to $250,300,325,350,375$, and 400 ${ }^{\circ} \mathrm{C}$ in $\mathrm{N}_{2}$, after which each sample was analyzed with HAADFSTEM and EDX (Figure S9). In every sample and for each temperature, four representative rods were analyzed with EDX to determine their core-to-shell ratios and their compositions, which were close to the average sample compositions, as given in Table 1. From the core-to-shell ratio, the degree of alloying was calculated in the same way as described for the in situ TEM measurements, and the degree of alloying is shown as a function of the heating temperature in Figure 4a. It shows that the $\mathrm{Au} @ \mathrm{Ag} @ \mathrm{SiO}_{2} \mathrm{NRs}$ with average $X_{\mathrm{Ag}}$ values of $0.17,0.46$, and 0.72 alloy at 305,345 , and $375^{\circ} \mathrm{C}$, respectively. The EDX maps in Figure S9 show that all Au@Ag@SiO ${ }_{2} \mathrm{NRs}$ of the same composition simultaneously convert from a core-shell to the alloyed state.

In Figure 4b, an overview of the alloying temperatures versus the $\mathrm{Ag}$ content for all three techniques is shown. The ex situ data nicely support the trends observed in the in situ TEM and in situ EXAFS measurements. In all three techniques, the alloying temperature increases with increasing Ag content, and only the absolute temperatures vary. The ex situ TEM measurements match the EXAFS results, but the alloying temperatures determined by in situ TEM are $75-90{ }^{\circ} \mathrm{C}$ too high. The relatively high alloying temperatures from the in situ TEM measurements could be related to an altered heat conductivity in the $\mathrm{SiN}_{X}$ chip after depositing the nanorods combined with possible carbon contamination, leading to inaccurate temperatures in the heating chip. Alternatively, the strongly reducing electron beam could have influenced the kinetics of the alloying process, but we did not observe significant differences in the alloying process between areas that were or were not illuminated with the electron beam prior to the heating. Thus, although care should be taken in deducing quantitative data from in situ TEM, it is a powerful technique in providing a qualitative insight in the metal redistribution for single nanoparticles and correctly shows the dependency of the metal redistribution on the metal composition for different nanoparticles.
For all $\mathrm{Au}-\mathrm{Ag}$ compositions, the observed alloying temperatures are far below the bulk melting point of $\mathrm{Au}$ and $\mathrm{Ag}$, which points at a nanosize effect on the alloying process and enhanced atom mobilities compared to the bulk. Size effects have been observed for the melting temperatures of nanoparticles, where the melting point was significantly lowered when decreasing the nanostructure size. ${ }^{34-36}$ Analogously, the observed lowering of the alloying temperature can be explained by a lowering in cohesive energy, which is the binding strength of the atom with its neighbors, with increasing particle surface to volume ratio. ${ }^{37,38}$ Because the cohesive energy is proportional to the vacancy formation energy and activation energy of diffusion, it is to be expected that the mobility of atoms and the rate of alloying increases for smaller nanostructures, leading to lower alloying temperatures. When decreasing the particle diameter below $5 \mathrm{~nm}$, even spontaneous alloying of bimetallic $\mathrm{Au}-\mathrm{Ag}$ nanoparticles at room temperature can occur. ${ }^{39}$

It should be noted that the observed trend between the alloying temperature and metal composition in the $\mathrm{Au}-\mathrm{Ag}$ nanoparticles varies oppositely to the composition dependency in the melting point temperature, where $T_{\text {melting,Ag }}=962$ and $T_{\text {melting,Au }}=1064{ }^{\circ} \mathrm{C}$. A similar trend for atomic diffusion was measured in bulk Au-Ag crystals. ${ }^{40}$ The activation energy of diffusion for both $\mathrm{Au}$ and $\mathrm{Ag}$ atoms in $\mathrm{Au}-\mathrm{Ag}$ alloys was reported to increase with increasing Ag fraction going from 1.74 to $1.93 \mathrm{eV}$ for $\mathrm{Ag}$ atoms in pure $\mathrm{Au}$ and $\mathrm{Ag}$ and 1.81 to $2.09 \mathrm{eV}$ for $\mathrm{Au}$ atoms in pure $\mathrm{Au}$ and $\mathrm{Ag} .{ }^{40}$ From these activation energies, it follows that $\mathrm{Ag}$ atoms are more mobile than $\mathrm{Au}$ atoms but that the diffusion of both $\mathrm{Au}$ and $\mathrm{Ag}$ atoms is slower in high-Ag-content $\mathrm{Au}-\mathrm{Ag}$ alloys. A possible explanation for this phenomenon can be derived from the energy of vacancy formation and atom migration, which are known to be higher in $\mathrm{Ag}$ compared to Au: $E$ (vacancy formation $)_{\mathrm{Ag}}=1.10 \mathrm{eV}, E(\text { vacancy formation })_{\mathrm{Au}}=0.97 \mathrm{eV}$, $E$ (atom migration $)_{\mathrm{Ag}(\mathrm{in}) \mathrm{Ag}}=0.83 \mathrm{eV}$, and $E$ (atom migratio$\mathrm{n})_{\mathrm{Ag}(\mathrm{in}) \mathrm{Au}}=0.77 \mathrm{eV} .^{4-42}$ Because atomic diffusion in $\mathrm{Au}-\mathrm{Ag}$ crystals is known to go via vacancy-hopping, a lower number of vacancies and a higher energy cost for hopping into the vacancies with increasing $\mathrm{Ag}$ content could explain the observed retardation of $\mathrm{Au}$ and $\mathrm{Ag}$ in high-content $\mathrm{Ag}$ alloys. In addition to $\mathrm{Au}-\mathrm{Ag}$, similar trends of self-diffusion dependency opposite to melting temperature have been reported for, e.g., $\mathrm{Ag}-\mathrm{Mn},{ }^{43} \mathrm{Ti}-\mathrm{Cr},{ }^{44,45}$ and $\mathrm{Tl}-\mathrm{Pb}$ bulk 
a

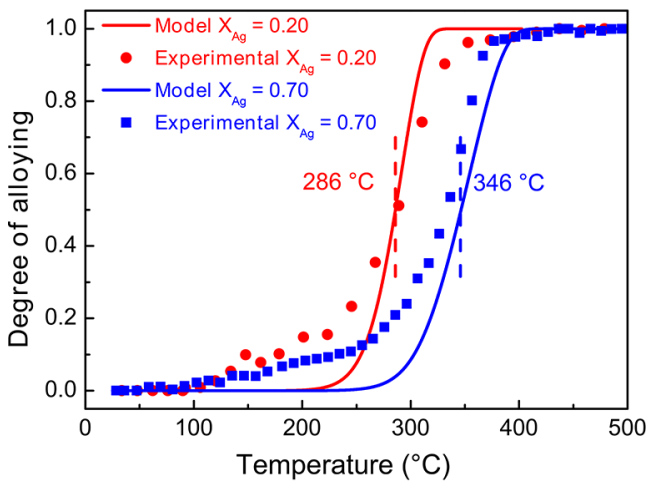

\section{b Theoretical prediction Ag dependence}

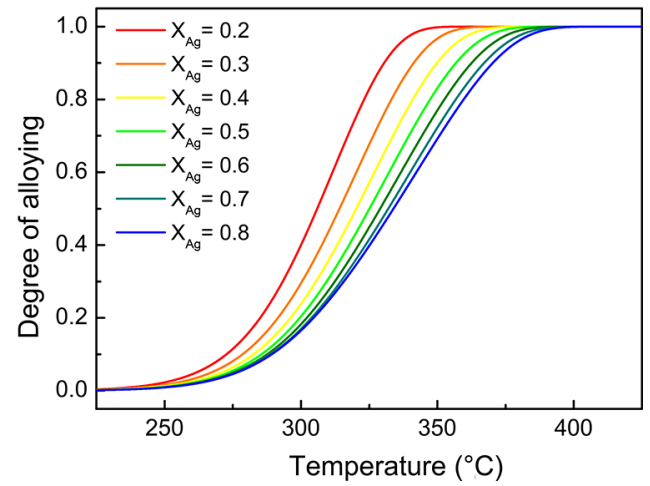

Figure 5. Theoretical prediction of the change in the degree of alloying as a function of heating temperature and atomic Ag fraction. (a) The theoretical prediction for the in situ EXAFS experiment give an alloying temperature of 286 and $346{ }^{\circ} \mathrm{C}$ for the samples with $X_{\mathrm{Ag}}=0.20$ and 0.70. The plot in panel $b$ shows the theoretical prediction of the alloying curves for $\mathrm{Au}-\mathrm{Ag} \mathrm{NRs}$ of $V=4 \times 10^{4} \mathrm{~nm}^{3}$ and $X_{\mathrm{Ag}}=0.2-0.8$ (from red to blue) heated with $3{ }^{\circ} \mathrm{C} / \mathrm{min}$.

crystals. ${ }^{46}$ In this study, we show experimental proof of this trend at the nanoscale.

Modeling Atomic Redistribution. We devised a simple model that can correctly describe the diffusion in $\mathrm{Au}-\mathrm{Ag}$ nanoparticles as a function of temperature and composition. We numerically calculate the diffusion of $\mathrm{Au}$ atoms $n_{\mathrm{Au}}$ and $\mathrm{Ag}$ atoms $n_{\mathrm{Ag}}$ passing through a static $\mathrm{Au}-\mathrm{Ag}$ interface per time step $\Delta t$ according to Fick's first law:

$$
n_{\mathrm{Ag}} / \Delta t=(A / r) \times D_{0}^{\mathrm{Ag}} e^{-Q_{\mathrm{Ag}} /(R T)} \times\left|C_{\text {core }}^{\mathrm{Ag}}-C_{\text {shell }}^{\mathrm{Ag}}\right|
$$

where $A$ is the interface area, $r$ the radius of the NP, $\mathrm{D}_{0}^{\mathrm{Ag}}$ the frequency factor, $Q_{\mathrm{Ag}}$ the activation energy, $R$ the gas constant, $T$ the temperature, $C_{\text {core }}^{\mathrm{Ag}}$ the silver concentration of the core, and $C_{\text {shell }}^{\mathrm{Ag}}$ the silver concentration of the shell (expressed in atoms per cubic meter). An analogous formula holds for the $\mathrm{Au}$ atoms. The rate of diffusion was calculated iteratively, where $D_{0}, Q$ and the concentration difference $C_{\text {core }}-C_{\text {shell }}$ were updated every time step. The frequency factor $D_{0}$ and activation energy for diffusion $Q$ depend on the $\mathrm{Au}-\mathrm{Ag}$ composition and have been measured experimentally in bulk crystals. ${ }^{40}$ We corrected these composition-dependent bulk $D_{0}$ and $Q$ values for the NP size according to the model of Guisbiers et al. ${ }^{4748}$ Herein, the activation energy of diffusion in NPs $Q_{N P}$ was derived from the activation energy of diffusion in the bulk $Q_{\text {bulk }}$ by using a so-called shape factor $\alpha_{\text {shape }}$ which, among others, depends on the surface-to-volume ratio of the NPs. More details on the calculation of the $\alpha_{\text {shape }}$ factor can be found in the Experimental section. For the $\mathrm{Au}-\mathrm{Ag}$ NRs used in this study, the correction of $Q_{\text {bulk }}$ to $Q_{\mathrm{NP}}$, resulted in alloy temperatures of $\sim 50{ }^{\circ} \mathrm{C}$ lower compared with bulk crystals of the same $\mathrm{Au}-\mathrm{Ag}$ composition.

The resulting theoretical predictions for the alloying curves of the in situ EXAFS measurements are shown in Figure 5a. The theoretical predictions are in very good agreement with the experimental in situ EXAFS data, and the alloying temperatures as predicted by the model, 286 and $346{ }^{\circ} \mathrm{C}$ for the $X_{\mathrm{Ag}}=0.20$ and 0.70 , respectively, match the experimental values of 287 and $334{ }^{\circ} \mathrm{C}$ closely. In Figure $5 \mathrm{~b}$, we show the theoretical prediction for the alloying curves of $\mathrm{Au}-\mathrm{Ag} \mathrm{NRs}$ with $V=4 \times 10^{4} \mathrm{~nm}^{3}$ and $X_{\mathrm{Ag}}=0.2-0.8$. In the calculation of these curves a heating ramp of $3{ }^{\circ} \mathrm{C} / \mathrm{min}$ was considered, as in the experimental studies. The theoretical predictions clearly demonstrate the importance of including the dependency of the diffusion on the metal composition. We stress that it is remarkable that the metal composition still plays such a crucial role in the diffusion kinetics in nanoparticles, in which size and shape have generally been considered to play the mostimportant role, ${ }^{12,33,47}$ and the influence of the particle composition has therefore been neglected so far.

\section{CONCLUSIONS}

We have used a multitechnique approach to precisely follow metal redistribution, a process crucial in catalysis, in situ, and at different length scales. A combination of in situ TEM with in situ EXAFS validated with ex situ measurements provided both a single particle and ensemble-averaged characterization. Our well-defined model system, consisting of mesoporous silicacoated, single-crystalline Au-core Ag-shell nanorods of tunable size and composition, allowed a systematic study of the nanoparticle composition on the atomic redistribution kinetics. We unambiguously showed that the atomic diffusion in $\mathrm{Au}-$ $\mathrm{Ag}$ nanoparticles strongly depends on the composition, a trend that has been observed in bulk crystals but that has, to the best of our knowledge, not been reported for nanomaterials. Additionally, we find indications for a nanoscale effect on the alloying process, leading to lower alloying temperatures when decreasing the nanoparticle size. Finally, we show that to correctly model metal redistribution in metallic nanoparticles, not only the nanoscale dimensions but also the metal composition should be taken into account. Both our experimental approach and the theoretical model are likely to apply to a wide range of bimetallic nanoparticle-based materials.

\section{EXPERIMENTAL SECTION}

Chemicals. All chemicals were used as received without further purification. Hexadecyltrimethylammonium bromide (CTAB, $>98.0 \%$ ) and sodium oleate $(\mathrm{NaOL},>97.0 \%)$ were purchased from TCI America. Hydrogen tetrachloroaurate trihydrate $\left(\mathrm{HAuCl}_{4} \cdot 3\right.$ $\mathrm{H}_{2} \mathrm{O}$ ) and sodium hydroxide (98\%) were purchased from Acros Organics. L-Ascorbic Acid (BioXtra, $\geq 99 \%)$, silver nitrate $\left(\mathrm{AgNO}_{3}, \geq\right.$ $99 \%)$, sodium borohydride $\left(\mathrm{NaBH}_{4}, 99 \%\right)$, hydrochloric acid $(\mathrm{HCl}$, 37 wt \% in water), tetraethyl orthosilicate (TEOS, 98\%), and ammonium hydroxide solution ( $\geq 25$ wt $\%$ in water) were purchased from Sigma-Aldrich. Ultrapure water (Millipore Milli-Q grade) with a resistivity of $18.2 \mathrm{M} \Omega$ was used in all of the experiments. All glassware for the AuNR synthesis was cleaned with fresh aqua regia $(\mathrm{HCl} /$ 
$\mathrm{HNO}_{3}$ in a 3:1 volume ratio), rinsed with large amounts of water and dried at $100{ }^{\circ} \mathrm{C}$ before usage.

Synthesis of the Au-Ag Nanorods. A total of three batches of $\mathrm{Au} @ \mathrm{Ag} @ \mathrm{SiO}_{2} \mathrm{NRs}$ with average $\mathrm{Ag}$ atomic fractions of 0.20, 0.46, and 0.70 were prepared by changing both the Au-core size and the Ag-shell thickness. The synthesis of the AuAg core-shell rods consists of four steps: AuNR synthesis (1), mesoporous silica coating (2), partial etching of AuNRs within their mesoporous silica shells (3), and Ag-shell growth on the etched AuNRs (4).

In the first step, monodisperse AuNRs were synthesized according to the protocol of Ye et al. ${ }^{49}$ A pair of $500 \mathrm{~mL}$ scale syntheses were carried out with growth solutions containing $7.0 \mathrm{~g}$ of CTAB, $1.24 \mathrm{~g}$ of $\mathrm{NaOL}, 25 \mathrm{~mL}$ of $\mathrm{MQ} \mathrm{H}_{2} \mathrm{O}$, and $250 \mathrm{~mL}$ of $1.0 \mathrm{mM} \mathrm{HAuCl}_{4} ; 7.2 \mathrm{~mL}$ of $\mathrm{AgNO}_{3} ; 2.1 \mathrm{~mL}$ of concentrated $\mathrm{HCl} ; 64 \mathrm{mM}$ ascorbic acid; and $1.0 \mathrm{~mL}$ of seed solution. The seeds were prepared from $10 \mathrm{~mL}$ of 0.10 M CTAB, $51 \mu \mathrm{L}$ of $\mathrm{HAuCl}_{4}$ and $1.0 \mathrm{~mL}$ of $\mathrm{NaBH}_{4}$. The subsequent rod growth was performed under static conditions in a $30{ }^{\circ} \mathrm{C}$ water bath overnight. The resulting AuNR suspensions had an absorption maximum of 4.0 at $\lambda($ LSPR $)=866$ and $853 \mathrm{~nm}$. The rods were centrifuged at $8000 \mathrm{rcf}$ for $30 \mathrm{~min}$ (Rotina 380R Hettich centrifuge), washed with $\mathrm{H}_{2} \mathrm{O}$, and redispersed in $5.0 \mathrm{mM} \mathrm{CTAB} \mathrm{H}_{2} \mathrm{O}$.

In the second step, the CTAB stabilized AuNRs were coated with a $18 \mathrm{~nm}$ mesoporous silica shell via the method of Gorelikov et al. ${ }^{32}$ The coating was performed in $350 \mathrm{~mL}$ of $1.5 \mathrm{mM} \mathrm{CTAB}$ aqueous solution containing $1.0 \mathrm{mM} \mathrm{NaOH}$ and an AuNR concentration corresponding to a absorption maximum of 10. During magnetic stirring at $300 \mathrm{rpm}$ in a $30^{\circ} \mathrm{C}$ water bath, 3 times $1.05 \mathrm{~mL}$ of $20 \mathrm{vol} \%$ TEOS in EtOH were added with a 30 min time interval. The Au@ $\mathrm{SiO}_{2} \mathrm{NRs}$ were centrifuged at $8000 \mathrm{rcf}$ for $30 \mathrm{~min}$ and washed with water and ethanol.

The third step, oxidative etching of the $\mathrm{Au} @ \mathrm{SiO}_{2} \mathrm{NRs}$, was performed by following the procedure described by Deng et al. ${ }^{9}$ but with $\mathrm{H}_{2} \mathrm{O}_{2}$ as an oxidant instead of $\mathrm{O}_{2}$ from air. Different core sizes were obtained by varying the etching time. For the rods with $X_{\mathrm{Ag}}=$ $0.20,240 \mathrm{~mL}$ of AuNRs in $\mathrm{MeOH}(\mathrm{Abs}=6.0)$ were heated to $60^{\circ} \mathrm{C}$ in an oil bath while magnetically stirring at $400 \mathrm{rpm}$ with $4.8 \mathrm{~mL}$ of $\mathrm{HCl}(37 \%)$ and $4.8 \mathrm{~mL}$ of $\mathrm{H}_{2} \mathrm{O}_{2}$ (0.2 wt \%). The LSPR peak position changed from 838 to $822 \mathrm{~nm}$ after etching for $10 \mathrm{~min}$. The reaction was quenched by putting the mixture in a $4{ }^{\circ} \mathrm{C}$ water bath and diluting it with $200 \mathrm{~mL}$ of ice-cold $\mathrm{MeOH}$ before centrifugation at $10000 \mathrm{rcf}$ for $20 \mathrm{~min}$. The etched rods were washed with and redispersed in EtOH. For batches with $X_{\mathrm{Ag}}=0.46$ and $0.70,210 \mathrm{~mL}$ of AuNRs in $\mathrm{MeOH}, 4.8 \mathrm{~mL}$ of $\mathrm{HCl}(37 \%)$, and $4.8 \mathrm{~mL}$ of $\mathrm{H}_{2} \mathrm{O}_{2}(0.2$ wt \%) were added. After 13 and $26 \mathrm{~min}, 100 \mathrm{~mL}$ of reaction mixture was quenched with $100 \mathrm{~mL}$ of ice-cold $\mathrm{MeOH}$ and was as described above. The LSPR peak positions of the rods were 750 and $694 \mathrm{~nm}$.

Finally, the procedure by Deng et al. was modified to do the $\mathrm{Ag}$ overgrowth in large reaction volumes $(\gg 1 \mathrm{~mL}) . \mathrm{HCl}$ was added to lower the Ag reduction rate by ascorbic acid and allows for a homogeneous shell growth on all particles. The rods with $X_{\mathrm{Ag}}=0.20$ were prepared by adding $2.0 \mathrm{~mL}$ of $0.1 \mathrm{M} \mathrm{HCl}, 3.0 \mathrm{~mL}$ of $5.0 \mathrm{mM}$ $\mathrm{AgNO}_{3}$, and $3.0 \mathrm{~mL}$ of $20 \mathrm{mM}$ ascorbic acid were added to $200 \mathrm{~mL}$ of aqueous AuNR suspension $(\mathrm{Abs}=4.5, \mathrm{LSPR}=780 \mathrm{~nm})$ while stirring vigorously. The rods with $X_{\mathrm{Ag}}=0.70$ were prepared in 2 steps. To 120 $\mathrm{mL}$ of rod suspension was added $1.2 \mathrm{~mL}$ of $0.1 \mathrm{M} \mathrm{HCl}, 6.6 \mathrm{~mL}$ of 5.0 $\mathrm{mM} \mathrm{AgNO}_{3}$, and $6.6 \mathrm{~mL}$ of $20 \mathrm{mM}$ ascorbic acid. After washing with $\mathrm{MQ} \mathrm{H}_{2} \mathrm{O}$, a second Ag-overgrowth step was performed to increase the Ag content. To $100 \mathrm{~mL}$ of aqueous Au@Ag@SiO 2 NR suspension $($ Abs $=1.2$, LSPR $=701 \mathrm{~nm}), 1.0 \mathrm{~mL}$ of $0.1 \mathrm{M} \mathrm{HCl}, 4.0 \mathrm{~mL}$ of 5.0 $\mathrm{mM} \mathrm{AgNO}$, and $4.0 \mathrm{~mL}$ of $20 \mathrm{mM}$ ascorbic acid were added. The $X_{\mathrm{Ag}}=0.46$ sample was prepared on a smaller scale because it was only used for the ex situ and in situ TEM measurements. To $1.0 \mathrm{~mL}$ of aqueous $\mathrm{Au} @ \mathrm{SiO}_{2} \mathrm{NRs}$ suspension $(\mathrm{Abs}=2.5, \mathrm{LSPR}=745 \mathrm{~nm}), 10$ $\mu \mathrm{L}$ of $0.1 \mathrm{M} \mathrm{HCl}, 40 \mu \mathrm{L}$ of $5.0 \mathrm{mM} \mathrm{AgNO}$, and $40 \mu \mathrm{L}$ of $20 \mathrm{mM}$ ascorbic acid were added.

All Au@Ag@SiO $\mathrm{S}_{2}$ NRs were washed with $\mathrm{MQ} \mathrm{H}_{2} \mathrm{O}$ and ethanol, redispersed in ethanol, and stored at $4{ }^{\circ} \mathrm{C}$ to prevent oxidation and dissolution of the $\mathrm{Ag}$ shell. The centrifugation speed varied between 6000 and $8000 \mathrm{rcf}$ depending on the volume of the rods. The samples were dried at $60{ }^{\circ} \mathrm{C}$ in air. All samples were characterized with visible-near-infrared spectroscopy and TEM.

In Situ TEM. The in situ heating measurements were carried out on a FEI Talos F200X operated at $200 \mathrm{kV}$ using a heating holder from DENSsolutions. A mix of four different batches of $\mathrm{Au} @ \mathrm{Ag} @ \mathrm{SiO}_{2} \mathrm{NRs}$ was drop-cast on a heating chip (Wildfire Nanochip) with silicon nitride windows. The overall heating ramp was set to $3{ }^{\circ} \mathrm{C} / \mathrm{min}$. EDX maps were acquired at $25,250,300$, and $350{ }^{\circ} \mathrm{C}$ and from 400 to 650 ${ }^{\circ} \mathrm{C}$ with a $25{ }^{\circ} \mathrm{C}$ temperature interval. The acquisition time per EDX map was $5 \mathrm{~min}$, and the probe current was $700 \mathrm{pA}$. In the intervals between the EDX acquisitions, the beam was blanked to minimize the influence of the electron beam on the alloying process. Different SiN windows were checked during heating that were not illuminated prior to heating. No significant differences in alloying kinetics were observed between the illuminated and non-illuminated spots. The $\mathrm{SiN}$ chip was plasma was cleaned for $10 \mathrm{~s}$ in a $20 \% \mathrm{O}_{2}$ in an $\mathrm{Ar}$ atmosphere before the TEM experiment.

In Situ EXAFS. The in situ EXAFS measurements were performed at the ROCK beamline of the SOLEIL synchrotron. At this beamline, continuous switching between the $\mathrm{Au} \mathrm{L}_{3}$ edge $(11919 \mathrm{eV})$ and the $\mathrm{Ag}$ $\mathrm{K}$ edge $(25514 \mathrm{eV})$ is possible (time to switch $\sim 1 \mathrm{~min}$ ) using two Quick-XAS monochromators equipped with $\mathrm{Si}(111)$ and $\mathrm{Si}(220)$ channel-cut crystals, respectively. The operation parameters of the monochromators were set to record two EXAFS spectra per second. The powdered samples were loaded in a stainless steel sample holder (with a depth of $1 \mathrm{~mm}$ ) allowing temperature control and gas flow. The $X_{\mathrm{Ag}}=0.20$ and $0.70 \mathrm{Au} @ \mathrm{Ag} @ \mathrm{SiO}_{2} \mathrm{NR}$ samples were diluted with boron nitride. The heating was done in a He flow of $25 \mathrm{~mL} / \mathrm{min}$ and with a heating rate of $3{ }^{\circ} \mathrm{C} / \mathrm{min}$. Before and after heating, EXAFS spectra were collected for $500 \mathrm{~s}$ at each edge at room temperature and averaged. During the temperature ramps, alternate measurements at both edges were performed continuously: spectra were collected and averaged for $35 \mathrm{~s}$ at the Au edge and 60 to $120 \mathrm{~s}$ at the Ag edge, depending on the quality of the Ag signal. Measurements were done in transmission mode using ionization chambers as detectors. Energy calibration was ensured by the simultaneous measurement of the absorption spectra of metallic $\mathrm{Au}$ and $\mathrm{Ag}$ foils.

Spectra analysis was conducted with the IFEFFIT library using the GUI Athena and Artemis. ${ }^{50}$ All spectra were energy calibrated to the first inflection point of the $\mathrm{Ag}$ or $\mathrm{Au}$ foil at 25514 and $11919 \mathrm{eV}$, respectively. EXAFS signal was extracted in Athena with a $R=1.0$ cutoff and a $k$ weight of 2 and Fourier transformed using a Hanning window in $k=3$ and $\mathrm{d} k=1$. EXAFS analysis was conducted in Artemis with the normalized spectra exported from Athena. The amplitude reduction factor $\left(S_{0}^{2}\right)$ of 0.83 for $\mathrm{Ag}$ and 0.79 for $\mathrm{Au}$ was obtained by fitting the EXAFS data of the respective metal foils. The simulation of scattering paths for the bi-metallic samples was performed with the ATOMS algorithm with a custom input file created by substituting $\mathrm{Au}$ atoms by $\mathrm{Ag}$ in the first shell to obtain the closest rational fraction of atoms. A correction factor was introduced to $S_{0}^{2}$ to obtain the actual sample composition. Structural parameters were determined by multiple $k$-weight least-squares fitting, and the goodness of fit was determined by observing the reduced $\chi^{2}$ and $R^{2}$ statistical parameters. The linear combination fitting was carried out in Athena on the normalized $\mu(E)$ spectra in the region between -20 to $120 \mathrm{eV}$ from the absorption edge.

Ex situ Heating. The ex situ heating experiments were performed in a tubular oven (Thermolyne 79300 tube furnace) under a constant $\mathrm{N}_{2}$ flow. The three different samples were each drop-cast on a copper TEM grid (200 mesh copper (100), Formvar/carbon film) and heated in a ceramic cup placed in a quartz oven tube. The heating rate was always $3{ }^{\circ} \mathrm{C} / \mathrm{min}$, and the particles were heated to $250,300,325$, 350,375 , and $400{ }^{\circ} \mathrm{C}$ and cooled under $\mathrm{N}_{2}$ to room temperature before taking them out of the oven. After heating, all samples were analyzed with HAADF and EDX on a FEI Talos F200X, operated under the same conditions as described above.

Diffusion Model. To predict the rate of alloying in $\mathrm{Au}-\mathrm{Ag}$ nanoparticles, we numerically calculated Fick's first law. The number of $\mathrm{Au}, n_{\mathrm{Au}}$, and $\mathrm{Ag}$ atoms, $n_{\mathrm{Ag}}$, diffusing through the static $\mathrm{Au}-\mathrm{Ag}$ interface per time step was calculated with eq 2 . After each step, the 
Au-to-Ag ratio of the core and the shell was updated, affecting $D_{0}, Q$ $C_{\text {core }}$, and $C_{\text {shell }}$ in eq 2. The values for $D_{0}$ and $Q$ for silver and gold diffusing into various $\mathrm{Au}-\mathrm{Ag}$ compositions were taken from the work of Mallard et al. ${ }^{40}$ These bulk values of $D_{0}$ and $Q$ were corrected for NP size effects according to the model of Guisbiers, ${ }^{47,48}$ in which the change in activation energy for a NP compared to the activation energy in the bulk can be described as:

$$
\frac{Q_{\mathrm{NP}}}{Q_{\text {bulk }}}=1-\frac{\alpha_{\text {shape }}}{D}
$$

where the shape factor $\alpha_{\text {shape }}$ is given by: $:^{47}$

$$
\alpha_{\text {shape }}=\frac{D\left(\gamma_{s}-\gamma_{l}\right)}{\Delta H_{m, \infty}} \times \frac{S}{V}
$$

Here, $D$ is the diameter of the NP; $\gamma_{s, l}$ are the surface energies in the solid and the liquid phases, respectively; $S$ the surface area of the NP; $V$ the volume of the NP; and $\Delta H_{\mathrm{m}, \infty}$ the bulk melting enthalpy.

Lastly, the temperature was updated every time step according to the temperature ramp used in the experiments. Usually, one time step was $1 \mathrm{~s}$, which ensured small changes in the $\mathrm{Au}-\mathrm{Ag}$ content per time step for the temperatures used in this work. Subsequent time steps were evaluated until the core and shell consist of the same $\mathrm{Au}-\mathrm{Ag}$ composition, when a full alloy composition is reached. Only geometric input parameters determined from TEM such as the core-shell volume, the interface and surface area and the radius of the NP were needed for the calculations.

\section{ASSOCIATED CONTENT}

\section{S Supporting Information}

The Supporting Information is available free of charge on the ACS Publications website at DOI: 10.1021/acsnano.8b03978.

Figures showing EDX maps, optical spectra of the different batches of $\mathrm{Au} @ \mathrm{Ag} @ \mathrm{SiO}_{2}$ NRs, high-resolution TEM images, the analysis method that was used to deduce the degree of alloying from the EDX maps, additional alloying curves derived from the in situ TEM measurements, additional EXAFS spectra, and alloying curves. Tables showing the fitting parameters used to calculate the coordination numbers from the EXAFS data. (PDF)

\section{AUTHOR INFORMATION}

\section{Corresponding Authors}

*E-mail: A.vanBlaaderen@uu.nl.

*E-mail: P.E.deJongh@uu.nl.

\section{ORCID}

Petra E. de Jongh: 0000-0002-2216-2620

\section{Notes}

The authors declare no competing financial interest.

\section{ACKNOWLEDGMENTS}

The authors thank Dr. M.A. van Huis for providing the in situ electron microscopy heating equipment, $M$. Bransen for useful discussion on the nanorod synthesis, and Dr. S. Dussi for critically reading the manuscript. This project has received funding from the European Research Council (ERC) under the European Union's Horizon 2020 research and innovation programme (ERC-2014-CoG no. 648991) and the ERC under the European Unions Seventh Framework Programme (FP2007-2013) and ERC Advanced Grant Agreement no. 291667 HierarSACol. J.v.d.H. also acknowledges the Graduate programme of the Debye Institute for Nanomaterials Science (Utrecht University), which is facilitated by the grant
022.004.016 of the NWO, The Netherlands Organisation for Scientific Research. The authors acknowledge the award of beamtime on the ROCK beamline at Synchrotron SOLEIL under proposal no. 20151175. The work on ROCK beamline was supported by a public grant overseen by the French National Research Agency (ANR) as a part of the "Investissements d'Avenir" program (ref no. ANR-10-EQPX-45).

\section{REFERENCES}

(1) Gilroy, K. D.; Ruditskiy, A.; Peng, H.-C.; Qin, D.; Xia, Y. Bimetallic Nanocrystals: Syntheses, Properties, and Applications. Chem. Rev. 2016, 116, 10414-10472.

(2) de Aberasturi, D. J.; Serrano-Montes, A. B.; Liz-Marzán, L. M. Modern Applications of Plasmonic Nanoparticles: From Energy to Health. Adv. Opt. Mater. 2015, 3, 602-617.

(3) Ferrando, R.; Jellinek, J.; Johnston, R. L. Nanoalloys: From Theory to Applications of Alloy Clusters and Nanoparticles. Chem. Rev. 2008, 108, 845-910.

(4) Singh, A. K.; Xu, Q. Synergistic Catalysis over Bimetallic Alloy Nanoparticles. Chem CatChem 2013, 5, 652-676.

(5) Liu, X.; Wang, A.; Yang, X.; Zhang, T.; Mou, C. Y.; Su, D. S.; Li, J. Synthesis of Thermally Stable and Highly Active Bimetallic Au-Ag Nanoparticles on Inert Supports. Chem. Mater. 2009, 21, 410-418.

(6) Sun, K. Q.; Hong, Y. C.; Zhang, G. R.; Xu, B. G. Synergy between $\mathrm{Pt}$ and $\mathrm{Au}$ in Pt-on-Au Nanostructures for Chemoselective Hydrogenation Catalysis. ACS Catal 2011, 1 (10), 1336-1346.

(7) Hong, J. W.; Kim, D.; Lee, Y. W.; Kim, M.; Kang, S. W.; Han, S. W. Atomic-Distribution-Dependent Electrocatalytic Activity of Au-Pd Bimetallic Nanocrystals. Angew. Chem., Int. Ed. 2011, 50, 8876-8880.

(8) Albrecht, W.; Van Der Hoeven, J. E. S.; Deng, T. S.; De Jongh, P. E.; van Blaaderen, A. Fully Alloyed Metal Nanorods with Highly Tunable Properties. Nanoscale 2017, 9, 2845-2851.

(9) Deng, T. S.; Van Der Hoeven, J. E. S.; Yalcin, A. O.; Zandbergen, H. W.; Van Huis, M. A.; Van Blaaderen, A. Oxidative Etching and Metal Overgrowth of Gold Nanorods within Mesoporous Silica Shells. Chem. Mater. 2015, 27, 7196-7230.

(10) Gao, C.; Hu, Y.; Wang, M.; Chi, M.; Yin, Y. Fully Alloyed Ag/ $\mathrm{Au}$ Nanospheres: Combining the Plasmonic Property of Ag with the Stability of Au. J. Am. Chem. Soc. 2014, 136, 7474-7479.

(11) Bonifacio, C. S.; Carenco, S.; Wu, C. H.; House, S. D.; Bluhm, H.; Yang, J. C. Thermal Stability of Core-Shell Nanoparticles: A Combined In Situ Study by XPS and TEM. Chem. Mater. 2015, 27, 6960-6968.

(12) Lasserus, M.; Schnedlitz, M.; Knez, D.; Messner, R.; Schiffmann, A.; Lackner, F.; Hauser, A. W.; Hofer, F.; Ernst, W. E. Thermally Induced Alloying Processes in a Bimetallic System at the Nanoscale: AgAu sub-5 nm Core-Shell Particles Studied at Atomic Resolution. Nanoscale 2018, 10, 2017-2024.

(13) Pramanik, S.; Chattopadhyay, S.; Das, J. K.; Manju, U.; De, G. Extremely Fast Au-Ag Alloy-Dealloy Associated Reversible Plasmonic Modifications in $\mathrm{SiO}_{2}$ Films. J. Mater. Chem. C 2016, 4, 3571-3580.

(14) Hwang, B. J.; Sarma, L. S.; Chen, J. M.; Chen, C. H.; Shih, S. C.; Wang, G. R.; Liu, D. G.; Lee, J. F.; Tang, M. T. Structural Models and Atomic Distribution of Bimetallic Nanoparticles as Investigated by X-ray Absorption Spectroscopy. J. Am. Chem. Soc. 2005, 127, $11140-11145$.

(15) Ding, Y.; Fan, F.; Tian, Z.; Wang, Z. Atomic Structure of Au-Pd Bimetallic Alloyed Nanoparticles. J. Am. Chem. Soc. 2010, 132, 12480-12486.

(16) Masoud, N.; Delannoy, L.; Calers, C.; Gallet, J. J.; Bournel, F.; de Jong, K. P.; Louis, C.; de Jongh, P. E. Silica-Supported Au-Ag Catalysts for the Selective Hydrogenation of Butadiene. ChemCatChem 2017, 9, 2418-2425.

(17) Zugic, B.; Wang, L.; Heine, C.; Zakharov, D. N.; Lechner, B. A. J.; Stach, E. A.; Biener, J.; Salmeron, M.; Madix, R. J.; Friend, C. M. Dynamic Restructuring Drives Catalytic Activity on Nanoporous Gold-Silver Alloy Catalysts. Nat. Mater. 2016, 16, 558-565. 
(18) Destro, P.; Kokumai, T. M.; Scarpellini, A.; Pasquale, L.; Manna, L.; Colombo, M.; Zanchet, D. The Crucial Role of the Support in the Transformations of Bimetallic Nanoparticles and Catalytic Performance. ACS Catal. 2018, 8, 1031-1037.

(19) Sandoval, A.; Aguilar, A.; Louis, C.; Traverse, A.; Zanella, R. Bimetallic Au- $\mathrm{Ag} / \mathrm{TiO}_{2}$ Catalyst Prepared by Deposition- Precipitation: High Activity and Stability in CO Oxidation. J. Catal. 2011, $281,40-49$.

(20) Tao, F.; Salmeron, M. In Situ Studies of Chemistry and Structure of Materials in Reactive Environments. Science 2011, 331, 171-174.

(21) Xin, H. L.; Alayoglu, S.; Tao, R.; Genc, A.; Wang, C. M.; Kovarik, L.; Stach, E. A.; Wang, L. W.; Salmeron, M.; Somorjai, G. A.; Zheng, $\mathrm{H}$. Revealing the Atomic Restructuring of Pt-Co Nanoparticles. Nano Lett. 2014, 14, 3203-3207.

(22) Holse, C.; Elkjær, C. F.; Nierhoff, A.; Sehested, J.; Chorkendorff, I.; Helveg, S.; Nielsen, J. H. Dynamic Behavior of CuZn Nanoparticles Under Oxidizing and Reducing Conditions. J. Phys. Chem. C 2015, 119, 2804-2812.

(23) Tao, F.; Grass, M. E.; Zhang, Y.; Butcher, D. R.; Renzas, J. R.; Liu, Z.; Chung, J. Y.; Mun, B. S.; Salmeron, M.; Somorjai, G. A. Reaction-Driven Restructuring of Rh-Pd and Pt-Pd Core-Shell Nanoparticles. Science 2008, 322, 932-934.

(24) Ahmadi, M.; Behafarid, F.; Cui, C.; Strasser, P.; Cuenya, B. R. Long-Range Segregation Phenomena in Shape-Selected Bimetallic Nanoparticles: Chemical State Effects. ACS Nano 2013, 7, 91959204.

(25) Carenco, S.; Wu, C. H.; Shavorskiy, A.; Alayoglu, S.; Somorjai, G. A.; Bluhm, H.; Salmeron, M. Synthesis and Structural Evolution of Nickel-Cobalt Nanoparticles Under $\mathrm{H}_{2}$ and $\mathrm{CO}_{2}$. Small 2015, 11 , 3045-3053.

(26) Hodak, J. H.; Henglein, A.; Giersig, M.; Hartland, G. V. LaserInduced Inter-Diffusion in AuAg Core-Shell Nanoparticles. J. Phys. Chem. B 2000, 104, 11708-11718.

(27) Liao, H.; Fisher, A.; Xu, Z. J. Surface Segregation in Bimetallic Nanoparticles: A Critical Issue in Electrocatalyst Engineering. Small 2015, 11, 3221-3246.

(28) Sarkar, R.; Rentenberger, C.; Rajagopalan, J. Electron Beam Induced Artifacts During In Situ TEM Deformation of Nanostructured Metals. Sci. Rep. 2015, 5, 1-11.

(29) Woehl, T. J.; Evans, J. E.; Arslan, I.; Ristenpart, W. D.; Browning, N. D. Direct In Situ Determination of the Mechanisms Controlling Nanoparticle Nucleation and Growth. ACS Nano 2012, 6, 8599-8610.

(30) Van Den Berg, R.; Elkjaer, C. F.; Gommes, C. J.; Chorkendorff, I.; Sehested, J.; De Jongh, P. E.; De Jong, K. P.; Helveg, S. Revealing the Formation of Copper Nanoparticles from a Homogeneous Solid Precursor by Electron Microscopy. J. Am. Chem. Soc. 2016, 138, 3433-3442.

(31) Frenkel, A. I. Applications of Extended X-ray Absorption FineStructure Spectroscopy to Studies of Bimetallic Nanoparticle Catalysts. Chem. Soc. Rev. 2012, 41, 8163-8178.

(32) Gorelikov, I.; Matsuura, N. Supporting Information Single-Step Coating of Mesoporous Silica on CTAB- Capped Nanoparticles. Nano Lett. 2008, 8, 369-373.

(33) Guisbiers, G.; Mendoza-Cruz, R.; Bazán-Dız, L.; VelázquezSalazar, J. J.; Mendoza-Perez, R.; Robledo-Torres, J. A.; RodriguezLopez, J. L.; Montejano-Carrizales, J. M.; Whetten, R. L.; JoséYacamán, M. Electrum, the Gold-Silver Alloy, From the Bulk Scale to the Nanoscale: Synthesis, Properties, and Segregation Rules. ACS Nano 2016, 10, 188-198.

(34) Dick, K.; Dhanasekaran, T.; Zhang, Z.; Meisel, D. SizeDependent Melting of Silica-Encapsulated Gold Nanoparticles. J. Am. Chem. Soc. 2002, 124, 2312-2317.

(35) Buffat, P.; Borel, J.-P. Size Effect on the Melting Temperature of Gold Partlcles. Phys. Rev. A: At., Mol., Opt. Phys. 1976, 13, 22872298.

(36) Castro, T.; Reifenberger, R.; Choi, E.; Andres, R. P. SizeDependent Melting Temperature of Individual Nanometer-Sized
Metallic Clusters. Phys. Rev. B: Condens. Matter Mater. Phys. 1990, 42, $8548-8556$

(37) Yang, C. C.; Li, S. Investigation of Cohesive Energy Effects on Size-Dependent Physical and Chemical Properties of Nanocrystals. Phys. Rev. B: Condens. Matter Mater. Phys. 2007, 75, 165413.

(38) Nanda, K. K.; Sahu, S. N.; Behera, S. N. Liquid-Drop Model for the Size-Dependent Melting of Low-Dimensional Systems. Phys. Rev. A: At., Mol., Opt. Phys. 2002, 66, 013208.

(39) Shibata, T.; Bunker, B. A.; Zhang, Z.; Meisel, D.; Vardeman, C. F.; Gezelter, J. D. Size-Dependent Spontaneous Alloying of Au-Ag Nanoparticles. J. Am. Chem. Soc. 2002, 124, 11989-11996.

(40) Mallard, W.; Gardner, A. B.; Bass, R.; Slifkin, L. Self-Diffusion in Silver-Gold Solid Solutions. Phys. Rev. 1963, 129, 617-625.

(41) Doyama, M.; Koehler, J. S. Quenching and Annealing of Lattice Vacancies in Pure Silver. Phys. Rev. 1962, 127, 21-31.

(42) Simmons, R. O.; Balluffi, R. W. Measurement of Equilibrium Concentrations of Lattice Vacancies in Gold. Phys. Rev. 1962, 125, $862-872$.

(43) Hagel, W.; Westbrook, J. Silver Diffusion in the Intermetallic Compound AgMg. Trans. TMS-AIME 1961, 221, 951.

(44) Mortlocks, A. J.; Tomlm, D. H. The Atomic Diffusion of Chromium in the Titanium-Chromium System. Philos. Mag. 1959, 4, 628-643.

(45) Peart, R.; Tomlin, D. Diffusion of Solute Elements in BetaTitanium. Acta Metall. 1962, 10, 123-134.

(46) Resing, H.; Nachtmeb, N. Self-Diffusion of Lead, Thallium and Bismuth in the Solid Lead-Thallium System. J. Phys. Chem. Solids 1961, 21, 40-56.

(47) Guisbiers, G.; Buchaillot, L. Modeling the Melting Enthalpy of Nanomaterials. J. Phys. Chem. C 2009, 113, 3566-3568.

(48) Guisbiers, G. Size-Dependent Materials Properties Toward a Universal Equation. Nanoscale Res. Lett. 2010, 5, 1132-1136.

(49) Ye, X.; Zheng, C.; Chen, J.; Gao, Y.; Murray, C. B. Using Binary Surfactant Mixtures To Simultaneously Improve the Dimensional Tunability and Monodispersity in the Seeded Growth of Gold Nanorods. Nano Lett. 2013, 13, 765-771.

(50) Ravel, B.; Newville, M. ATHENA, ARTEMIS, HEPHAESTUS: Data Analysis for X-Ray Absorption Spectroscopy using IFEFFIT. J. Synchrotron Radiat. 2005, 12, 537-541. 